\title{
Diagnóstico de las variables que inciden en la implementación de un modelo hacia la Educación Superior 4.0
}

\author{
Diagnostic of the variables that affect the implementation of a model towards \\ Higher Education 4.0
}

GALVÁN-MORALES, Patricia†*, MEDINA-TORRES, Ma. Guadalupe, VILLALÓN-GUZMÁN, Maria Teresa y ASATO-ESPAÑA, Julio Armando

Tecnológico Nacional de México en Celaya

ID $1^{\mathrm{er}}$ Autor: Patricia, Galván-Morales / ORC ID: 0000-0002-2619-6115, CVU CONACYT ID: 441943

ID $1^{\text {er }}$ Coautor: Ma. Guadalupe, Medina-Torres / ORC ID: 0000-0001-6617-1812, CVU CONACYT ID: 64685

ID $2^{\text {do }}$ Coautor: Maria Teresa, Villalón-Guzmán / ORC ID: 0000-0001-5595-474X, CVU CONACYT ID: 368722

ID $3^{\text {er }}$ Coautor: Julio Armando, Asato-España / ORC ID: 0000-0002-7464-2335, CVU CONACYT ID: 503061

DOI: $10.35429 /$ JTAE.2019.8.3.9.15

Recibido: 04 de Marzo, 2019; Aceptado 30 de Junio, 2019

\begin{abstract}
Resumen
Uno de los requisitos principales de la llamada cuarta revolución industrial o industria 4.0 (I4.0), es el recurso humano preparado, innovador y con una actitud de adaptación, por ello las Instituciones de Educación Superior (IES) enfrentan el desafío de ofrecer una nueva generación de profesionistas que participen y promuevan dicha transformación. Es de suma importancia la formación de recursos humanos competentes ante los retos que implican el desarrollo e incorporación de diferentes estrategias para el proceso de migración de los procesos industriales, ahora tradicionales, hacia las pautas marcadas por la cuarta revolución industrial. La presente investigación es un diagnóstico que permite identificar y evaluar las competencias requeridas por la I4.0 y la forma en que las IES deberán adecuar su quehacer educativo. En este contexto se consideraron cinco ejes rectores involucrados; los actores, la infraestructura disponible, la vinculación, la gestión y el I+D+i (Investigación, Desarrollo tecnológico e innovación), de manera que permita la adaptación curricular en esa dirección. El resultado permitirá proponer un modelo de evaluación para la Educación Superior 4.0.
\end{abstract}

Diagnóstico, Educación Superior, Industria 4.0

\begin{abstract}
One of the main requirements of the so-called fourth industrial revolution or industry 4.0 (I4.0), is the human resource prepared, innovative and with an attitude of adaptation, for that reason the Higher Education Institutions (HEI) face the challenge to offer a new generation of professionals who participate and promote this transformation. It is very important to train competent human resources in the face of the challenges involved in the development and incorporation of different strategies for the migration process of industrial processes, now traditional, towards the guidelines set by the fourth industrial revolution. The present investigation is a diagnostic that allows to identify and evaluate the competences required by the I 4.0 and the way in which the HEIs should adapt their educational work. In this context, five guiding axes were considered involved; the actors, the available infrastructure, the relationships, the management and the $\mathrm{R}+\mathrm{D}+\mathrm{i}$ (Research, Develpment and innovation), in a way that allows the curricular adaptation in that direction. The result will allow proposing an evaluation model for Higher Education 4.0.
\end{abstract}

Diagnostic, Higher Education, Industry 4.0

Citación: GALVÁN-MORALES, Patricia, MEDINA-TORRES, Ma. Guadalupe, VILLALÓN-GUZMÁN, Maria Teresa y ASATO-ESPAÑA, Julio Armando. Diagnóstico de las variables que inciden en la implementación de un modelo hacia la Educación Superior 4.0. Revista de Tecnología y Educación. 2019. 3-8: 9-15

\footnotetext{
* Correspondencia del Autor: (patricia.galvan@itcelaya.edu.mx)

$\dagger$ Investigador contribuyendo como primer autor.
} 


\section{Introducción}

La Industria 4.0 (I4.0) es el resultado de la evolución y transformación de la revolución Industrial, la cual se presenta como una realidad y desafío de las economías emergentes para cerrar la brecha tecnológica que tienen con los países desarrollados. Muchas propuestas al respecto de la I4.0 se han presentado como una revolución, lo que "implica un cambio completo de las estructuras" (Usatorre \& Pedrero, 2015), donde se plantea entre muchas otras ideas la Fabrica del Futuro, que demanda en el presente profesionistas y profesionales preparados en procesos y diseños inteligentes, relación hombre-máquina y naves industriales pqueñas, flexibles y "ecológicas"

(Manufactura, 2018).

Por otro lado las instituciones educativas son un actor importante en esta evolución y transformación, ya que es esencial capacitar a los ciudadanos del siglo XXI en dirección a esta tendencia, para lo cual es necesario implementar cambios que propicien el desarrollo de las competencias requeridas actualmente. La innovación en educación tienen ante sí como principal reto los procesos de adopción por parte de las personas, los grupos y las instituciones.

En la historia, el impacto de los medios de comunicación ha sido radical para modificar la conducta y en general la forma de vivir, pues las distancias y los tiempos se hicieron más pequeños con el internet, que además contienen un nuevo patrón, que es la cantidad invaluable de información que puede obtenerse a partir de datos obtenidos automáticamente desde muy diversos orígenes. Sin excluir a los medios que posibilitan este hecho, pues ellos también presentan una amplia gama informática, sino que con internet, la posibilidad de obtener información está ahí, y permanece en el tiempo, sin embargo, para que esto sea una realidad es preciso afrontar un enorme reto: la desinformación.

Al igual que como ocurrió con los medios de comunicación, todos los niveles educativos deben estar preparados para recibir y poder manejarse dentro de esta nueva era. Adultos, jóvenes y niños necesitan prepararse para las condiciones económicas y sociales que las nuevas tecnologías traen consigo (Díaz, 2012).
En este contexto, el Tecnológico Nacional de México (TecNM) en Celaya, institución de educación superior, ante los retos que se plantean está el dar respuesta a las demandas del entorno digitalizado y transformar su modelo educativo para adaptarlo a las exigencias y demandas de la I4.0.

Es imprescindible considerar que uno de los más imperiosos desafíos a los que se enfrenta la educación superior, es responder a la necesidad ineludible de actualizar y mejorar las competencias tanto de estudiantes como de docentes. Las universidades deberán responder con mayor flexibilidad a los requerimientos cambiantes de la sociedad y especialmente a las necesidades individuales de este nuevo alumnado.

El TecNM en Celaya acepta el reto de formar una nueva generación de profesionistas a través del cual se busca consolidar las fortalezas institucionales relacionadas con el nuevo paradigma e integrarlas a la Educación Superior 4.0.

En este contexto, la idea es que el conocimiento se manifieste de manera innovadora en la propia institución y su entorno, mediante la gestión de un nuevo modelo educativo que propicie la formación de los futuros ingenieros y sume a la nueva generación de profesionales en la Industria 4.0.

A continuación se describen los antecedentes que sostienen el contexto a exponer, para posteriormente ubicar el modelo educativo 4.0 a través del diagnóstico de variables basados en los resultados de un proyecto de investigación realizado en el TecNM en Celaya.

\section{Antecedentes}

Más que una cuarta revolución tecnológica, actualmente está ocurriendo una revolución cultural. El desarrollo tecnológico invariablemente lleva a pensar en el futuro donde pueden converger todas las disciplinas y ámbitos del conocimiento, la investigación, la producción y la creatividad, desde la informática y la ingeniería hasta la filosofía, las ciencias sociales, el arte y la comunicación (Organización de las Naciones Unidas, 2015). 
El desarrollo tecnológico puede ofrecer oportunidades para alcanzar los objetivos establecidos en la Agenda 2030 de Naciones Unidas para el Desarrollo Sostenible. Por una parte, sus aplicaciones pueden propiciar el avance más rápidamente, permitiendo una mejor evaluación de riesgos, pronósticos más precisos, mayores intercambios de conocimientos, así como soluciones innovadoras en los campos de la educación, la salud, la ecología, el urbanismo o las industrias creativas, por mencionar algunos, mejorando los niveles de vida y el bienestar diario (Organización de las Naciones Unidas, 2015).

En el Segundo Foro Internacional en Innovación en Guanajuato en el 2018, se mencionó que la sociedad moderna se encuentra inmersa en procesos de transformación dinamizados por los cambios acelerados de las tecnologías digitales. (Organización de las Naciones Unidas para la Educación, la Ciencia y la Cultura, 2018)

La evolución y revolución no se detiene, la Varkey Foundation lo resalta en el "Global Teacher Prize" y ha generado diferentes productos de aprendizaje para profesores y estudiantes, “..., el espíritu de colaboración, la empatía y el trabajo en equipo están llamados a definir la educación del futuro". (Doucet, y otros, 2019)

El TecNM en Celaya evoluciona para formar y transformar a la sociedad, mediante la creación de un Modelo Educativo que dé respuesta a las necesidades actuales y tendencias de una sociedad del conocimiento con mayores exigencias.

\section{Modelo Educativo 4.0}

Para abordar el tema de la reinvención, en el TecNM en Celaya se habla tanto de la capacidad de desarrollo científico como de la habilidad para la innovación tecnológica. Para lograr la reinvención, se debe poner en juego y fomentar la innovación en la enseñanza y el aprendizaje, siempre basada en la investigación científica, a la par de promover nuevas formas de organización, nuevos métodos, nuevas tecnologías de la información y del aprendizaje.
En consecuencia la universidad del futuro tiene que formar científicos y tecnólogos para la renovación del círculo virtuoso de la innovación; la fórmula que debe promoverse en la universidad 4.0 es $\mathrm{F}+\mathrm{I}+\mathrm{D}+\mathrm{i}$ (Formación + Investigación + Desarrollo + innovación); formación profesional del futuro basada en la investigación para impulsar el desarrollo y la innovación científica y académica. (Pedroza Flores, 2018)

Actualmente, la tendencia de la enseñanza y el aprendizaje es hacia un modelo adaptativo. Los docentes se renuevan ante el uso cada vez más asimilado de la inteligencia mixta en el aula y fuera de ella. Considerando las experiencias mundiales de la renovación del proceso de enseñanza- aprendizaje y su vinculación con el círculo virtuoso de la innovación (ver Tabla 1), se hace patente que la universidad del futuro es un espacio que incuba soluciones para los desafíos reales del mundo (The New Media Consortium (NMC), 2017).

\begin{tabular}{|c|c|c|}
\hline \multicolumn{3}{|c|}{ Tendencia (plazo) } \\
\hline Largo & Mediano & Corto \\
\hline $\begin{array}{ll}\text { Avance de las } \\
\text { culturas } & \text { de } \\
\text { innovación } & \end{array}$ & $\begin{array}{lr}\text { Crecimiento } & \text { del } \\
\text { interés en } & \text { la } \\
\text { medición } & \text { del } \\
\text { aprendizaje } & \\
\end{array}$ & $\begin{array}{ll}\text { Diseños } & \text { de } \\
\text { aprendizaje } & \\
\text { híbrido } & \end{array}$ \\
\hline $\begin{array}{lr}\text { Enfoques } & \text { de } \\
\text { aprendizaje más } \\
\text { profundo }\end{array}$ & $\begin{array}{lr}\text { Rediseño de } & \text { los } \\
\text { espacios } & \text { de } \\
\text { aprendizaje } & \end{array}$ & $\begin{array}{l}\text { Aprendizaje } \\
\text { colaborativo }\end{array}$ \\
\hline
\end{tabular}

Tabla 1 Tendencias de la Enseñanza-Aprendizaje Fuente: The New Media Consortium (NMC), 2017

Cabe señalar que la tabla 1 expone los plazos:

Largo: implica el avance en la adopción de nuevas tecnologías en la educación superior en cinco o más años.

- Mediano: implica el avance en la adopción de nuevas tecnologías en la educación superior de los próximos tres a cinco años.

- Corto: implica el avance en la adopción de nuevas tecnologías en la educación superior en los próximos uno o dos años. 
Se pone de manifiesto que la enseñanza de la educación 4.0 se centra en la adquisición de las competencias del siglo XXI, especialmente de todas aquellas que no pueden desempeñar los robots: la creatividad, la comunicación asertiva, el trabajo en equipo, el pensamiento creativo, la innovación, la forja de redes de trabajo y de colaboración, la inteligencia emocional, la resiliencia, entre otros.

Es una realidad y es necesario que el conocimiento se manifieste de manera innovadora en la propia institución y su entorno, mediante la gestión de un nuevo modelo educativo que propicie la formación de los futuros ingenieros y sume a la nueva generación de profesionales en la I 4.0. Así, la formación de los estudiantes deberá centrarse en el desarrollo de competencias tanto genéricas como específicas. En la transformación del modelo educativo se deben considerar aspectos tales como:

Favorecer el aprendizaje de los estudiantes a través del desarrollo de proyectos con el uso de tecnología.

Propiciar la interacción constante entre alumnos y profesores, centrándose en la comunicación como principal vehículo para favorecer los procesos de enseñanza y aprendizaje.

Resolver problemas reales por medio del aprendizaje bajo el enfoque de competencias, a fin contextualizar los conocimientos.

Favorecer el aprendizaje activo, con la finalidad de que el alumno sea capaz de regular su proceso de aprendizaje a través del pensamiento estratégico.

Promover la creación de entornos de aprendizaje reales con la finalidad de favorecer el aprendizaje significativo.

Implementar el uso de las Tecnologías de la Información y Comunicación (TIC) como herramientas de acceso, organización, creación, difusión de contenidos, diseño de escenarios de aprendizaje además de selección y uso de herramientas tecnológicas que faciliten este proceso de cambio.

\section{Propuesta Modelo Educación 4.0}

Los cinco ejes rectores son la parte medular del modelo; por su parte los actores (docentes, investigadores, trabajadores de la administración, y por supuesto los estudiantes) que sumados a los empresarios aportarán al modelo las mejores estrategias de generación de solucione que, mediante los otros ejes la infraestructura disponible, la vinculación, la gestión y el I+D+i (Investigación, Desarrollo tecnológico e innovación).

La propuesta del modelo Educativo del TecNM en Celaya 4.0 se basa en los principales actores que se conjuntan para que los estudiantes sean Profesionistas 4.0 (ver figura 1).

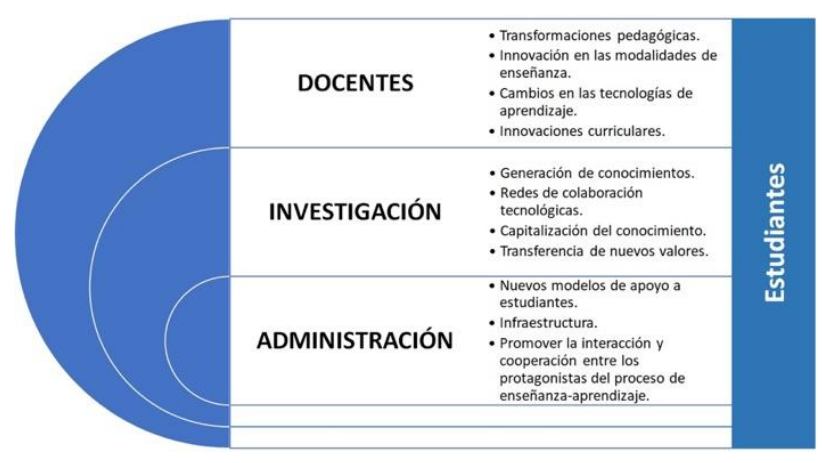

Figura 1 Modelo de Educación Superior 4.0

Fuente: Elaboración Propia

\section{Metodología a desarrollar}

El presente artículo se deriva de un proyecto de investigación desarrollado bajo un enfoque metodológico cuantitativo en donde se pretende relacionar ciertas fases conceptuales con los resultados de un proceso de recolección y análisis de datos, los cuales se estudiaron estadísticamente. Además es de tipo no experimental y transversal. No experimental porque no pretende manipular condiciones $\mathrm{o}$ incorporar estímulos a los sujetos de estudio, sino más bien recabar datos de su condición actual, y es de tipo transversal ya que hace una evaluación en un momento determinado (Hernández \& Mendoza, 2018).

Para dar el significado de las variables en redacción lineal, es importante la comparación de los criterios usados que se describen a continuación. 
La investigación se llevó a cabo a través de un diagnóstico con la finalidad de identificar y evaluar las competencias requeridas por la I4.0, para estudiantes, docentes y personal administrativo del TecNM en Celaya. Las preguntas que integraron el instrumento de recolección de datos consideraron los cinco ejes rectores involucrados; los actores, la infraestructura disponible, la vinculación, la gestión y el $\mathrm{I}+\mathrm{D}+\mathrm{i}$, de manera que permita identificar las necesidades de la adaptación curricular en esa dirección.

Se desarrolló un instrumento que consta de 60 cuestionamientos, desagregados entre preguntas para la caracterización del sujeto, el acopio de datos (vinculados a los ejes rectores) y algunos reactivos de verificación cruzada. El cuestionario se implementó mediante un formulario en la Web para facilitar su difusión y el acopio de datos.

Se realizó una prueba piloto para la validación del instrumento, aplicándose un muestreo estratificado por grupos de interés; estudiantes de licenciatura y posgrado, empleados docentes y administrativos, levantándose 72 cuestionarios durante los meses de marzo y abril del año 2019, que fueron validados aplicando el coeficiente de Alfa de Cronbach, obteniéndose un valor de 0.968, el cual corresponde un alto nivel de fiabilidad para el instrumento.

\section{Resultados}

Sobre los datos finales de la aplicación del instrumento, se iniciará por presentar los resultados de estadística descriptiva, comenzando por caracterizar la población encuestada, lo que corresponde al primer eje rector. Por sexo el $49 \%$ fueron mujeres y el $51 \%$ hombres; el $84 \%$ son estudiantes de licenciatura, el $7 \%$ de posgrado, un $7 \%$ es personal docente $\mathrm{y}$ un $2 \%$ personal administrativo o de apoyo a la educación.

Para el porcentaje de avance en la carrera para los estudiantes; el $47 \%$ están en el primer cuarto de la carrera, el $18 \%$ en el segundo y la misma proporción en el tercero, y solamente el $8 \%$ está en la última etapa de sus estudios (el restante 9\% corresponde a empleados, por lo que no aplica en este punto).
Los siguientes resultados corresponden a la medición de la importancia percibida que se da a diferentes competencias digitales, en el contexto de la I4.0.

Con relación a la infraestructura y tecnología, el segundo eje rector, la mayoría $(88 \%)$ reconoce que la institución ha proporcionado medios para el aprendizaje y dominio de las TICs, además de reconocer en proporciones cercanas o mayores al 50\% la importancia de poder generar, integrar, evaluar y aplicar información a nivel profesional. Respecto a la infraestructura el dominio de software, manejo de equipo computacional y en especial, el conocimiento y uso de redes de computadoras tienen una reconocida importancia. Respecto al tercer eje que corresponde a la vinculación, las habilidades blandas como la comunicación tienen una importancia dominante ya que obtuvo el $61 \%$ de relevancia, así como el conocimiento de aspectos legales (tales como la propiedad intelectual) también resultó con un alto nivel de reconocimiento por su importancia con un $49 \%$ de distinción.

Del cuarto eje, correspondiente a la gestión; la capacidad de planificación, organización, trabajo en equipo, adaptación al cambio, liderazgo y resolución de problemas resultaron ser los rasgos más reconocidos por su relevancia en el contexto de la I4.0, con más de un $50 \%$ de reconocimiento cada uno.

Finalmente sobre el análisis descriptivo, del quinto eje, que abarca el concepto $\mathrm{I}+\mathrm{D}+\mathrm{i}$, se encontró que la creatividad digital es relevante con un $76 \%$, el diseño de productos, el prototipado, la automatización y los microsistemas también resultaron con niveles destacados de significancia entre la comunidad tecnológica.

En el estudio de estadística inferencial se realizó un análisis correlacional bivariado aplicando el coeficiente de Pearson, en donde se encontró que variables como el desarrollo de software, el proceso de datos y la electrónica, cuentan con niveles de correlación elevados (superiores a 0.8 ) respecto a otras variables correspondientes a conceptos de la I4.0, en menor grado pero también por destacar se encuentran campos de conocimiento específicos como la realidad virtual y aumentada, el big data y la seguridad informática, que destacan en su correspondencia respecto a otras variables.

GALVÁN-MORALES, Patricia, MEDINA-TORRES, Ma. Guadalupe, VILLALÓN-GUZMÁN, Maria Teresa y ASATO-ESPAÑA, Julio Armando. Diagnóstico de las variables que inciden en la implementación de un modelo hacia la Educación Superior 4.0. Revista de Tecnología y Educación. 2019. 
Cabe decir que los resultados aquí expuestos son una síntesis de lo más relevante.

\section{Conclusiones}

Con los resultados obtenidos, puede concluirse que la comunidad del TecNM en Celaya está enterada de la existencia y relevancia de la I4.0, dado que aunque el $73 \%$ de los encuestados manifestó sólo tener conocimientos básicos o generales sobre el tema, el 98.6\% manifestó un alto interés en conocer más del concepto de I4.0.

Ahora bien, respecto al modelo de Educación Superior 4.0, en la tabla 2 se presenta para cada actor de este proceso, las variables de mayor interés y su nivel de desarrollo en la comunidad tecnológica.

\begin{tabular}{|l|l|l|}
\multicolumn{2}{|c}{ Actor } & \multicolumn{1}{c|}{$\begin{array}{c}\text { Nivel de } \\
\text { desarrollo }\end{array}$} \\
\hline Estudiantes & Aprendizaje de TICs & Medio \\
\hline & $\begin{array}{l}\text { Conocimientos de } \\
\text { electrónica }\end{array}$ & Bajo \\
\hline & Proceso de datos & Bajo \\
\hline & Sistemas mecatrónicos & Bajo \\
\hline & Prototipado & Bajo \\
\hline & Desarrollo de software & Bajo \\
\hline Docentes & Tecnologías digitales & Medio \\
\hline & Automatización & Medio \\
\hline & Creatividad digital & Bajo \\
\hline Investigadores & Inteligencia Artificial & Medio \\
\hline & Ciencia de datos & Medio \\
\hline & Propiedad intelectual & Medio \\
\hline Administraciónn & Redes de computadoras & Medio \\
\hline & Big Data & Bajo \\
\hline
\end{tabular}

Tabla 2 Resumen de las variables de mayor interés y su nivel de desarrollo en el TecNM en Celaya

Fuente: Elaboración Propia

Resulta evidente que el cambio de un paradigma de pensamiento en una comunidad es un reto formidable, pero si a eso se le añaden componentes tecnológicos, que corresponden a un proceso de planeación, implementación, operación y explotación de sistemas de información complejos y en tiempo real, la situación parece ser aún más complicada, sin embargo, la identificación de las variables y el nivel de desarrollo proporcionan un punto de partida a considerar, ya que la implementación de cualquier estrategia requiere de un primer paso, correspondiendo a responder a la pregunta: ¿por dónde hay que comenzar?

\section{Referencias}

Doucet, A., Evers, J., Guerra, E., Lopez, N., Soskil, M., \& Timmers, K. (2019). La enseñanza en la cuarta revolución industrial. Al borde del precipicio. México: Pearson Educación México.

Díaz C. L. (2012). Educación Virtual. México: Red Tercer Milenio. Recuperado de http://www.aliat.org.mx/BibliotecasDigitales/E ducacion/Educacion_virtual.pdf

Echeverría S., B., \& Martínez C., P. (2018). Revolución 4.0, Competencias, Educación y Orientación. Revista Digital de Investigación en Docencia Universitaria, Vol. 12 No. 2. Perú: Universidad Peruana de Ciencias Aplicadas. Recuperado de https://revistas.upc.edu.pe/index.php/docencia/a rticle/view/831

Hernández S., R., \& Mendoza T., Ch. P. (2018). Metodología de la investigación: las rutas cuantitativa, cualitativa y mixta. México: Mc. Graw Hill.

Manufactura (15 de enero de 2018). Así será la fábrica del futuro. Obtenido de manufactura.mx:

https://manufactura.mx/industria/2018/01/15/asi -sera-la-fabrica-del-futuro

Organización de las Naciones Unidas para la Educación, la Ciencia y la Cultura. (2018). Foro Internacional. Innovación, Educación Superior e Industria 4.0. Guanajuato, México: Autor.

Organización de las Naciones Unidas. (2015). Agenda 2030. México: Autor. Recuperado de http://www.onu.org.mx/agenda-2030/

Pedroza Flores, R. (2018). La universidad 4.0 con currículo inteligente 1.0 en la cuarta revolución industrial. Revista Iberoamericana para la Investigación y Desarrollo Educativo.

Usatorre, L., \& Pedrero, J. (2015). Industria 4.0: Un reto para la sociedad que construimos. Obtenido de MetalIndustria.com: https://www.metalindustria.com/media/uploads/ noticias/documentos/MI3_Tecnalia.pdf 
Tecnalia. (2014). Informe Fábrica del Futuro.

España: Autor. Recuperado de https://www.tecnalia.com/images/stories/Catalo gos/informe-fabrica-del-futuro.pdf

The New Media Consortium (NMC). (2017). Tendencias clave que aceleran la adopción de nuevas tecnologías en la educación superior. E.U.A.: Educause. Recuperado de https://bit.ly/2MQEIBi 